\title{
Kikuchi-Fujimoto Disease: A Case Report
}

\author{
Dr. Santa Naorem*, Dr. Vinodhkumar K.** ,Dr. Uttam Kumar Barman**, \\ Dr. Gautam Debbarma** Dr. M Amuba Singh*** \\ *Associate Professor, ** Post-graduate trainees, Department of Medicine, \\ Regional Institute of Medical Sciences, Imphal, ***Professor \& Head of the Department, Department of
} Biochemistry.

\begin{abstract}
Kikuchi-Fujimoto disease is a rare benign, self limiting disorder of unknown etiology characterized by regional, tender cervical lymphadenopathy and associated with mild fever. A 24 year old girl presented with mild to moderate fever and cervical lymphadenopathy for 3 weeks. Systemic examination was normal. Works up for sepsis, autoimmune diseases, lymphoproliferative disease were negative. Histopathological examination of the lymph node was consistent with Kikuchi's disease and there was self remission after 2 weeks of follow up. Its association with systemic lupus erythematosus makes it necessary to be aware of this condition and to follow up these patients and to consider as one of the differential diagnoses in young patients with cervical lymphadenopathy and fever of unknown origin. Treatment is symptomatic and spontaneous recovery occurs in 1 to 4 months. Awareness of this disease may help clinicians and pathologists to prevent misdiagnosis and inappropriate treatment.
\end{abstract}

Key-words: Lymphadenopathy, Kikuchi's disease

\section{Introduction:}

Kikuchi-Fujimoto disease is a rare benign clinico-pathological condition of unknown etiology presenting with fever and painful lymphadenitis ${ }^{1}$. It was first recognized by Kikuchi ${ }^{2}$ and Fujimoto ${ }^{3}$ independently in two separate cases among young women in 1972. It is also known as necrotizing histiocytic lymphadenitis. It is primarily a disease of young adult with mean age of 30 years and female preponderance. It is most commonly seen in Japan and other Asian countries and also seen in other parts of world ${ }^{4}$. AlKhawaja $\mathrm{S}$ et $\mathrm{al}^{5}$ also reported the first Kikuchi disease in Bahrain. Its association with systemic lupus erythematosus makes it necessary to be aware of this condition and follow up these patients ${ }^{1}$ and to consider as one of the differential diagnoses in young patients with cervical lymphadenopathy and fever of unknown origin. To my knowledge this is the first case from Eastern India and two cases have been reported from Southern India ${ }^{6,7}$.

\section{Case History:}

A 24 year old girl presented with history of fever for 3 weeks which was intermittent, mild to moderate degree, associated with mild headache and mild pain in the right side of the neck with swelling at the same site which is gradually progressive in size over the last 10 days. No history of anorexia, weight loss, nausea, rashes, arthralgia, oral ulcerations, contact with TB patient, respiratory or genitourinary symptoms or contact with animals or recent travel. On examination patient was afebrile, 5-6 cervical lymph nodes were palpable on the right side of the neck mainly posterior cervical chain, multiple, discrete, mobile, minimally tender and varying sizes, largest being $2 \times 3 \mathrm{~cm}$, with normal overlying skin. Rest of the general physical and systemic examination were normal.

On investigation: hemoglobin, $12.5 \mathrm{gm} \%$; total leukocyte count, 5840 cells/cumm; ESR, 50mm/ ${ }^{\mathrm{st}} \mathrm{hr}$; platelet count, 1,55,000/cumm; chest x ray was normal; Mantoux test, Widal test, HBsAg, HCV ab, HIV by ELISA were negative. Bone marrow examination was normal, ANA and anti dsDNA was negative, ultrasound abdomen showed mild hepatomegaly of $156 \mathrm{~mm}$. Histopathological examination of the lymph node (figure 1,2 \&3) was showing large mottled area suggestive of necrosis and foamy macrophages in sheets along with abundant karyorrhectic debris and cresentic histiocytes with eccentrically placed nucleus and engulfed debris consistent with Kikuchi's disease. Gram stain and Ziehl-Neelsen stain for acid fast bacilli were negative

\section{Discussion}

Kikuchi-Fujimoto disease was first reported in 1972 by Kikuchi $^{2}$ and Fujimoto ${ }^{3}$ et al, as lymphadenitis with focal proliferation of reticular cells associated with numerous histiocytes and extensive nuclear debris. The etiology and pathogenesis of Kikuchi's disease is not known but a viral ${ }^{7,8}$, bacterial like yersinia and protozoa infections are suggested ${ }^{1}$. A genetic predisposition has been noted and there is female preponderance with a mean age of $30 \mathrm{yrs}{ }^{4,9}$. It is a benign and self limiting condition with a low recurrence rate of $3-4 \%{ }^{10}$. It usually presents as fever of low grade which may persist for about one week, rarely up to a month along with lymph 
node involvement usually localized (cervical), painful, moderately enlarged $(1-2 \mathrm{~cm})$, occasionally much larger but usually not beyond $7 \mathrm{~cm}$ and sometimes as cutaneous manifestations like rashes, malar rash, erythematous papules. Other symptoms, like myalgia, arthralgia, chest pain and abdominal pain may also be present

Histopathological examination of lymph node is diagnostic and shows irregular paracortical areas of coagulative necrosis with abundant karyorrhectic debris distorting nodal architecture. Large number of different types of histiocytes at the margin of the necrotic areas. The karyorrhectic foci are formed predominantly by histiocytes and plasmacytoid monocytes, medium to large sized transformed lymphocytes (immunoblasts). Neutrophils are characteristically absent and plasma cells are either absent or scarce. CT scan of affected lymph node shows hypodense centre with peripheral ring enhancement corresponding to central necrosis ${ }^{11}$.

Because of lack of signs and symptoms and serological markers, diagnosis of Kikuchi's disease is based on histopathological findings. So early recognition of this disease helps in avoiding unnecessary investigations and treatment.

\section{Conclusion}

Kikuchi disease is benign and self limiting disorder and treatment is mainly symptomatic. Its association with systemic lupus erythematosus makes these patients to be followed up. Clinicians and pathologists should be aware of this disorder to prevent misdiagnosis and inappropriate treatment.

\section{References:}

[1] Kato A, Kono T, Ishii M, Wakasa K, Taniguchi S. Spontaneous clearance of psoriasis during the course of Kikuchi - Fujimoto disease. J Am Acad Dermatol 2002; 47: 287-88.

[2] Kikuchi M. lymphadenitis showing focal reticular cell hyperplasia with nuclear debris and phagocytes: A clinicopathological study.Acta Hematol Jpn 1972; 35: 379-80.

[3] Fujimoto Y, Kojima Y, Yamaguchi K. Cervical Subacute necrotising lymphadenitis; A new clinicopathological entity 1972; 20: 920-27.

[4] Dorfman RF, Berry GJ. Kikuchi's histiocytic necrotizing lymphadenitis: An analysis of 108 cases with emphasis on differential diagnosis. Semin Diagn Pathol 1988; 5: 329-45.

[5] AlKhwaja S, Khatoon A, Shameem S. Kikuchi's Disease. Bahrain Medical Bulletin 2006; 28(3).

[6] Malathi S, R Varadharajan, Shivbalan. Kikuchi’s disease: A case report. Indian Paediatrics 2004; 40: 192-94.

[7] Harris VK, Danda D, Murali NS, Das PK, Abraham M, Cherian AM, et al. Unusual association of kikuchi's disease and dengue virus infection evolving into systemic lupus erythematosus. J Indian Med Assoc 2000; 98: 391-93.

[8] Chiu CF, Chow KC, Lin TY. Virus infection in patients with histiocytic necrotizing lymphadenitis in Taiwan. Detection of EBV, type 1 human T cells lymphotropic virus and parvo virus B 19. Am J clin pathol 2000; 113: 774-81.

[9] Amir AR, Amir SS, Sheikh SS.Kikuchi Fujimoto Disease: Report of familial occurance in two HLA-identical non twin sisters, J Intern Med 2002; 252: 79-83.

[10] Dorfman RF. Histiocytic necrotising lymphadenitis of Kikuchi and Fujimoto. Arch Pathol Lab Med 1987; $111: 1026-29$.

[11] Tsang WYW, Chan JKC, Ng CS. Kikuchi's Lymphadenitis. A morphologic analysis of 75 cases with special reference to unusual features. Am J Surg Pathol 1994; 18: 872-76.

Fig. 1 Large mottled area suggestive of necrosis

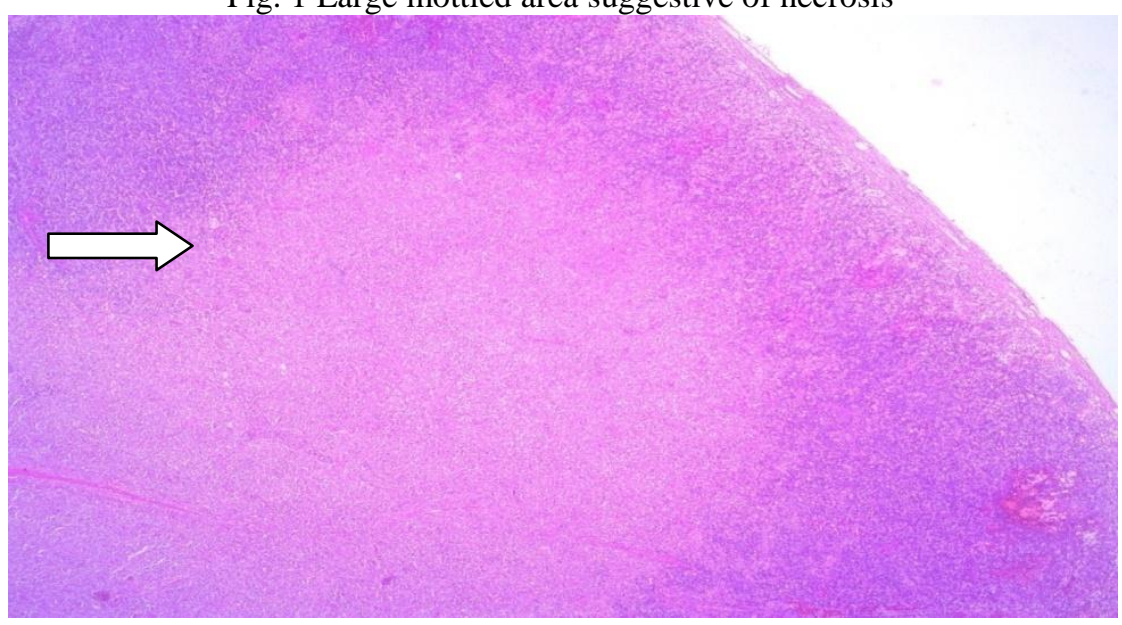


Fig. 2 Foamy macrophages in sheets with abundant karyorrhectic debris

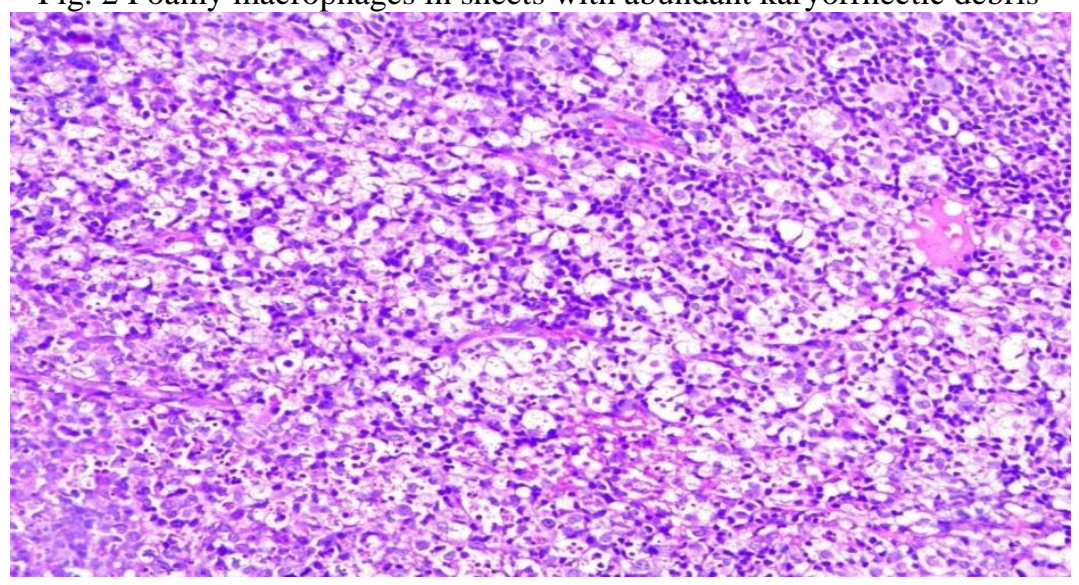

Fig.3 Crescentic histiocytes

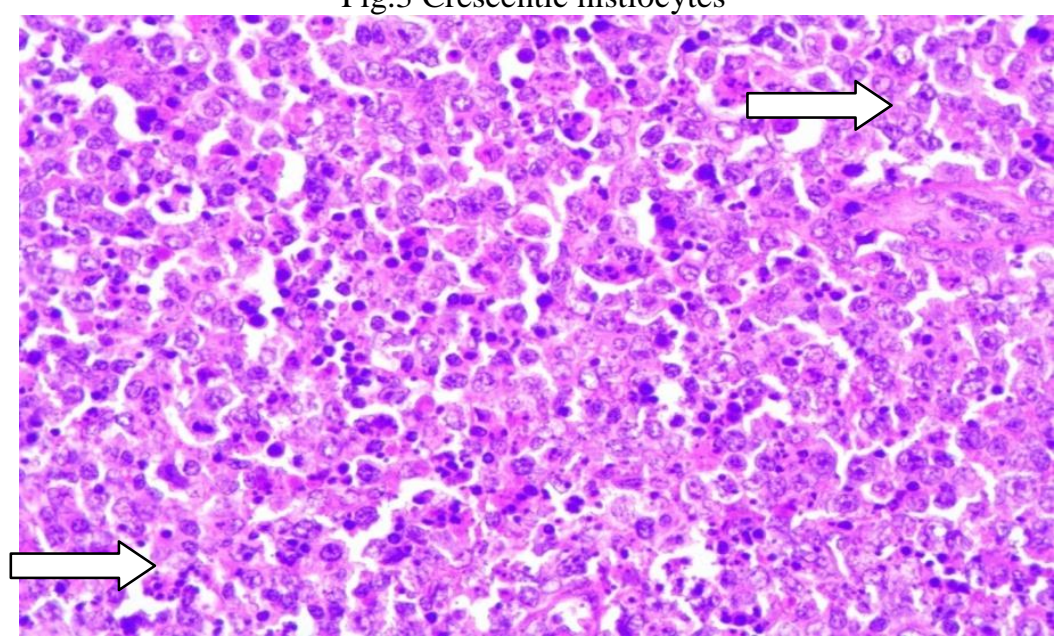

